\title{
La artesanía como estrategia pedagógica para la enseñanza de la identidad cultural ${ }^{1}$
}

\section{Crafts: a pedagogical strategy supported for the teaching of cultural identity}

DOI: http://dx.doi.org/10.17981/cultedusoc.9.3.2018.52

Artículo de investigación. Fecha de recepción: 15/06/2018. Fecha de aceptación: 27/11/2018

\author{
Frank Dussan Arquez; Carmen Mulford Palomino; Digna Machado Lopez; \\ Nelsy Machado Lopez; Maria Machado Martinez; Maria López Jimenez; \\ Lucia Arrazola Guerrero; Adria Lopez Simanca; Belky López Simanca; \\ Jaqueline Alvarino Badel; Samira Larios Castro; Nelsi Rivera Rivera; \\ Noralba Sequea Lara; Daniel Guzman Morales; \\ Boris Davila Machado y Ladys Luque Contrera ${ }^{2}$ \\ Institución Educativa Departamental Pijiño del Carmen
}

Para citar este artículo:

Dussan, F., Mulford, C., Machado, D., Machado, N., Machado, M., López M., Arrazola, L., López, A., López, B., Alvarino, J., Larios, S., Rivera, N., Sequea, N., Guzman, D., Davila, B. y Luque, L. (2018). La artesanía como estrategia pedagógica para la enseñanza de la identidad cultural. Cultura. Educación y Sociedad 9(3), 449-456. DOI: http://dx.doi.org/10.17981/cultedusoc.9.3.2018.52

\section{Resumen}

El propósito de la investigación se orienta al fomento de la identidad cultural de los estudiantes de la IED Pijiño del Carmen a través de la elaboración de Artesaníasa través del uso demateriales ecológicos del medio y las técnicas de utilizadas por los ancestros de la región. La unidad de análisis estuvo constituida por 100 estudiantes entre la edades de 11 a 16 años, los instrumentos utilizados para la recolección de información son la observación, y la encuesta. A nivel de resultados se halló que los estudiantes reconocen las artesanías propias de su contexto, que además su elaboración permite rescatar tradiciones y fomentar la cultura de su región. A partir de estos resultados se hace necesario impulsar la artesanía a fin de resaltar la relación de esta con la educación desde una comprensión cultural de los saberes tradicionales y de las prácticas sociales, que conforman el campo artesanal, por lo que se ha apostado a esta iniciativa a través de Estrategias Pedagógicas-EP.

Palabras clave: Artesanía, estrategia pedagógica, identidad cultural.

\section{Abstract}

The purpose of the research is to promote the cultural identity of students of the IED Pijiño del Carmen through the development of handicrafts through the use of environmental materials and techniques used by the ancestors of the region. The unit of analysis was constituted by 100 students between the ages of 11 to 16 years, the instruments used for the collection of information are observation, and the survey. At the level of results it was found that the students recognize the handicrafts proper to their context, which also allows them to rescue traditions and promote the culture of their region.From these results it is necessary to promote craftsmanship in order to highlight the relationship of this with education from a cultural understanding of traditional knowledge and social practices, which make up the artisanal field, which is why we have opted for this initiative through pedagogical strategies.

Keywords: Crafts, pedagogical strategy, cultural identity.

1 Este artículo ha sido derivado del Programa de Fortalecimiento de la Cultura Ciudadana y Democrática CT+I a través de la IEP apoyada en TIC en el Departamento de Magdalena: CICLON

2 Docentes miembros del grupo de investigación "Artesanos Pijiñeros".de la Institución Educativa Departamental Pijiño del Carmen

- The author; licensee Universidad de la Costa - CUC.

Cultura, Educación y Sociedad vol. 9 no. 3, pp. 449-456. Diciembre, 2018

Barranquilla. ISSN 2389-7724 Online 


\section{Introducción}

Es fundamental reconocer nuestra identidad cultural ya que los estudiantes del Institución EducativaTécnica Departamental (IETD)Pïiño del Carmen, no conocen las artesanías que se elaboran en su comunidad las cuales en su momento fueron muy importantes en las labores cotidianas, hoy en día se ha restado valor debido a la amplia gama de entretenimiento tecnológico anulando prácticamente el carácter cultural de este oficio partiendo del hecho que desde el curriculum no se implementa este tipo de actividades en el orden de los niveles de educación, ahora el área de artística hace referencia a nueve asignaturas que para el caso serían las manualidades, la danza, las artes plásticas, el dibujo, artes escénicas, entre otras; sin embargo, existen muchos factores que desmotivan la práctica de este tipo de trabajo escolar en los que se suman los costos, consecución de material y porque no tienen proyección económica sostenible según los estudiantes. Lo anterior, ha atraído como consecuencia que las futuras generaciones no tiene ninguna clase de conocimiento de los orígenes de este principio cultural.

Según UNESCO (2003), la importancia del patrimonio cultural inmaterial estriba en el acervo de conocimientos y técnicas que se transmiten de generación en generación. El conocimiento se origina en los grupos sociales y se transmitejunto a sus valores económicos, culturales e históricos, razón por la cual revisten la misma importancia para los países en su proceso de desarrollo.

Según lo anterior, el conocimiento que se tiene de las artesanías hasta llevarlo a las generaciones presentes y futuras no tiene otro carácter más que el de tradición técnica, donde los antepasados dejaron sus enseñanzas y a su propio estilo, precisamente surgieron como una necesidad ante la eventualidad. Esto no hace referencia a que las personas hayan subsistido económicamente con este arte, sino que simplemen- te los recursos existentes se lo permitían y daban rienda suelta a la creatividad, sin embargo, como hace parte de nuestras tradiciones, se quiere dar a conocer sobre este tipo de habilidades y destrezas a los jóvenes que muestran su interés, ya sea como aprenderlo como arte o simplemente por la información adquirida.

En Colombia, según el Artículo 2 del decreto 258 de 1987, la artesanía se entiende como "una actividad creativa y permanente de producción de objetos, realizada con predominio manual y auxiliada en algunos casos con maquinarias simples, obteniendo un resultado final individualizado, determinado por los patrones culturales, el medio ambiente y su desarrollo histórico".

La institución de alguna manera pretende evitar un caos cultural y se realizan mini ferias artesanales durante la semana cultural, que se realiza anualmente, pretendiendo involucrar al docente deemprendimiento para que se haga participe y logre incentivar a los estudiantes para que visionen este proyecto desde la perspectiva comercial y de esta forma controlar la extinción total del conocimiento en este campo. Por esto, lo que pretende este proyecto artesanos Pijiñeros, en su fundamento pedagógico, es extender esta actividad en todos los niveles escolares teniendo en cuenta las distintas habilidades por niveles; inquietando en este sentido el conocimiento en cuanto a la práctica artesanal, sus orígenes pero sobre todo conjugar el deseo por aprender, compañerismo, respeto, compromiso y responsabilidad por la identidad cultural propia de su región.

Cuando se lleva a cabo alguna investigación debe de tenerse en cuenta algunas características esenciales al seleccionarse la población bajo estudio, según Tamayo y Tamayo (1997) "la población se define como la totalidad del fenómeno a estudiar donde las unidades de la población posee una característica común la cual se estudia y da origen a los datos de la investigación" (p. 114). Por tal razón, la población de esta investigación son los estudiantes de la Ins- 
titución Educativa Técnica Departamental Pijiño del Carmen de preescolar, básica primaria, secundaria y media.

La apropiación del proyecto: "las artesanías: una estrategia pedagógica apoyada en las TIC para la enseñanza de la identidad cultural" es pertinente porque las nuevas generaciones tienen poco conocimiento de las artesanías en nuestro municipio y de su elaboración, principalmente por la población estudiantil de la Institución Educativa Técnica Departamental Pijiño del Carmen, se evidencia la necesidad de implementar un proyecto con el cual se motive a los jóvenes a la adquisición del conocimiento y apropiación de las técnicas de elaboración de las artesanías autóctonas, articulándolo en los procesos de enseñanza tomando como herramientas los conocimientos de los ancestros y el uso de las TIC.

A través de la transversalidad de las diferentes áreas, los estudiantes se apropiaran de las técnicas de elaboración de las artesanías mediante procesos investigativos y prácticos cuya finalidad será el rescate de nuestra identidad cultural.

El rescate de las artesanías será una estrategia motivadora en nuestra actividad pedagógica, para que el estudiante en forma creativa e innovadora elabore artesanías que le ayudaran a buscar recursos sostenibles en el tiempo.

Con este proyecto se busca resaltar la importancia de estudiar la artesanía en la educación, las artesanías tradicionales y sus expresiones son numerosas: herramientas, indumentarias, accesorios para festividades, recipientes, elementos empleados para el almacenamiento, arte decorativo y enseres domésticos. Los cuales pueden llegar a constituir un legado que se trasmite de generación en generación como ocurre en otras formas del patrimonio cultural inmaterial, muchas tradiciones artesanales cuentan con sistemas de instrucción y aprendizaje antiquísimos, está comprobado que al incentivar a nuestros estudiantes se convierte en un medio eficaz para reforzar y consolidar esos sistemas a través de ferias y muestras periódicas que hacen más atractiva la transferencia de conocimientos para ellos.

Dentro de las artesanías más representantes de la región encontramos:

\section{Escoba de paja}

De acuerdo con López (2011), son elaboradas con varitas de palma de vino, es una actividad artesanal tradicional de varias familias que han conservado el legado de sus ancestros, de esta actividad devengan en parte ingresos económicos. El producto final, o sea, las escobas, se comercializan en la región en donde tienen gran demanda por su utilidad y buenos precios.

La escoba artesanal es elaborada con la varita extraída de las hojas de la palma de vino, se extrae y se seca la varita, una vez seca es pesada y se forman montoncitos de una libra, posteriormente se tiende una pita de aproximadamente 1,20 metros de largo, para tejer la malla de la escoba.

\section{El Musengue}

Es un utensilio que se realiza del vástago de una planta llamada palma de vino, luego de un proceso adquiere semejanza como de un escobajo y se utiliza para sacudir moscas y mosquitos. Es de gran utilidad por esta región cenagosa, en donde por épocas abundan estos insectos.

\section{Las Totumas}

Son realizadas con la fruta del árbol del totumo y es utilizada para distintas actividades una de las cuales es el consumo de comidas y bebidas, además se usan con fines decorativos con un agregado de pinturas y grabados. Como conservación cultural es un utensilio que se utiliza en festividades realizadas en sitios campestres y en ocasiones especiales como semana santa para servir los dulces, mazamorra, chicha y sancochos propios de nuestra región. 


\section{Cucharas de Palo}

En la diversidad de especies del fruto del árbol de totumo encontramos una muy particular por su forma y tamaño, que permite a los artesanos explorar la creatividad e incluso a personas que no desarrollan este arte elaborar hermosas cucharas llamadas de palo.Son variados sus diseños y su utilidad es excepcional cuando se trata de disfrutar un delicioso plato de sopa o mazamorra caliente. Su elaboración consiste en cocinar el fruto, para luego sacar la semilla y después de un tiempo de secado utilizando cuchillo de sierra y lija se procede a elaborar las cucharas al gusto del artesano o de la persona que solicite el producto. En el mercado es pedido por docenas.

\section{La Esterilla}

Sustantivo femenino. Este vocabulario se refiere a un cairel o cinta hecho a base de oro y plata, por lo general muy estrecho. Tira o faja de esparto estrecho, también se puede elaborar mediante una paja de lienzo, tejido o tela de paja.

\section{La Investigación como Estrategia Pedagógica}

La propuesta de investigación referida al rescate y elaboración de artesanías propias del municipio de Pijiño del Carmen utilizando materiales ecológicos del medio y las técnicas de nuestros ancestros se convierte en una forma diferente de inducir en los niños el aprendizaje desde el momento en que se despierta el interés en aprender sobre algo a través de la investigación y la práctica. Desde este punto de vista, se procura un aprendizaje significativo como lo plantea Ausubel: "El aprendizaje es un proceso por medio del que se relaciona nueva información con algún aspecto ya existente en la estructura cognitiva de un individuo y que sea relevante para el material que se intenta aprender. El aprendizaje debe tener nece- sariamente, significado para el estudiante, si queremos que represente algo más que palabras o frases que repite de memoria en un examen".

Desde esta perspectiva la investigación en el aula permite que el niño indague, cuestione, analice, proponga y crea teniendo en cuenta los conocimientos previos, el contexto y su relación con los enfoques establecidos en el currículo institucional y sus componentes en transversalidad con las distintas áreas del conocimiento. Por consiguiente, la investigación como estrategia pedagógica es una herramienta que estimula el desarrollo de competencias y destrezas en lo referente al tema de investigación procurando que sea tan significativo hasta el punto de que se convierte en una necesidad para el mismo niño.

En el desarrollo de la IEP, el maestro es un guía dinamizador del proceso mediante el cual se integran los intereses del niño, los aportes pedagógicos y apropiación del uso de las TIC; fomentar la investigación en el aula es una estrategia que dinamiza el proceso educativo porque favorece la comunicación, el trabajo en equipo haciendo que la escuela se convierta en un escenario alejado de la rutina de las clases tradicionales porque va cambiando el modo de entender la investigación científica desde el punto de vista de la innovación desde el mundo de los estudiantes, los fines de la escuela y el método para lograrlo.

\section{Metodología}

Los instrumentos utilizados para la recolección de información de la muestra seleccionada en esta investigación son la observación, entrevista y la encuesta.

La observación: es el uso sistemático de nuestros sentidos en la búsqueda de los datos que necesitamos para resolver un problema de investigación, es directa cuando el investigador forma parte activa del grupo observado y asume sus comportamientos. 
La Encuesta: es semi-estructurada aplicada a una muestra de estudiantes que responderán a 15 preguntas. Grasso (2006) expresa que la encuesta permite explorar la opinión pública y los valores vigentes de una sociedad, temas de significación científica y de importancia en las sociedades democráticas.

Estas herramientas de indagación nos parecen apropiadas para obtener información pertinente en el nivel de importancia y aceptación de dicha propuesta.

\section{Resultados}

A través del análisis realizado a las herramientas aplicadas, se puede afirmar que la investigación de Pijiño del Carmen ha venido presentando cambios en los estudiantes, demostrandosu gentileza, humildad y disposición teniendo como condición humana el mantener sus tradiciones y costumbres. Entre las posibles causas que se cree han generado esta situación tenemos las experiencias al conocer cómo se realizan y los conocimientos de la población a estudiar.

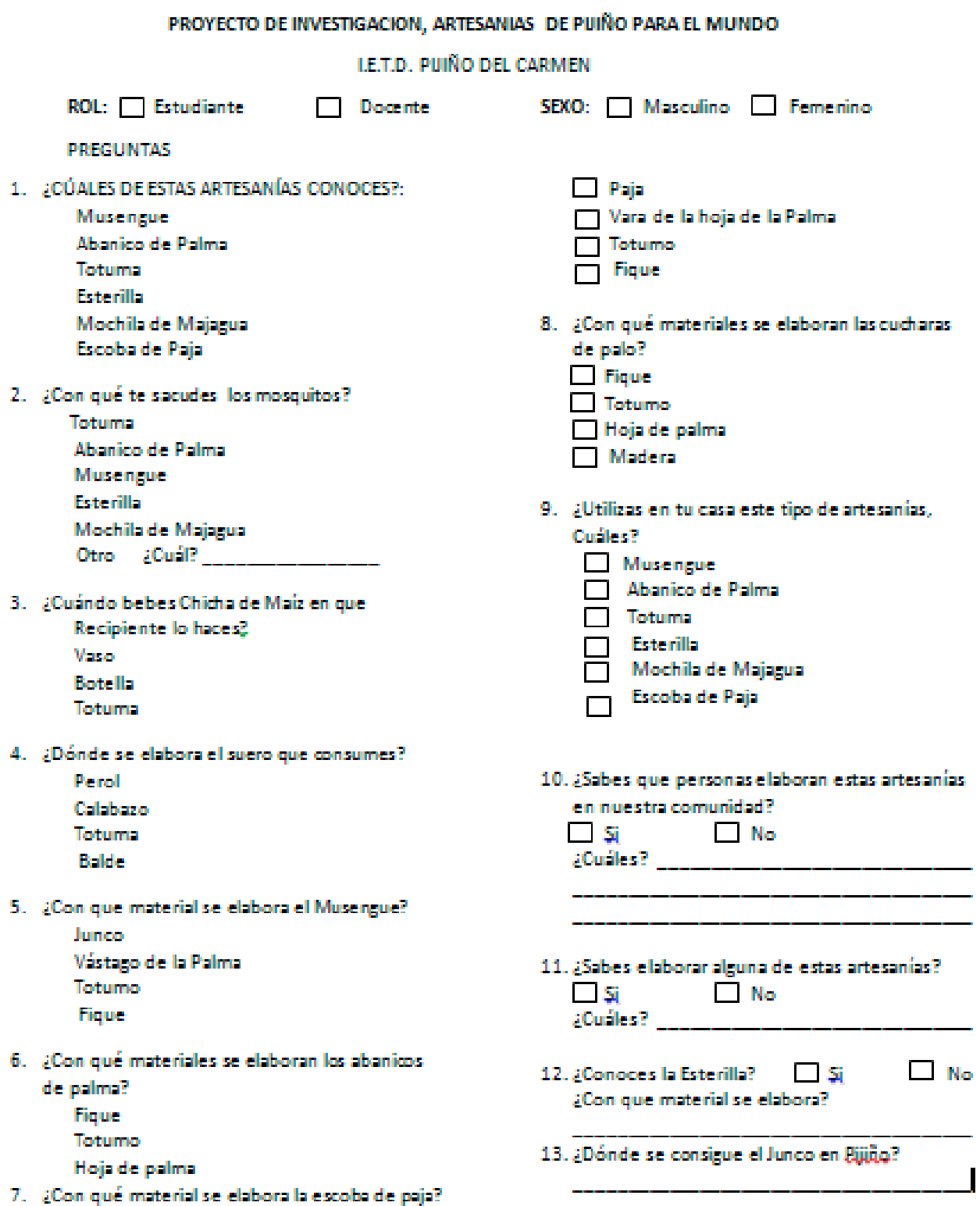




\section{¿CÚALES DE ESTAS ARTESANÍAS CONOCES?}

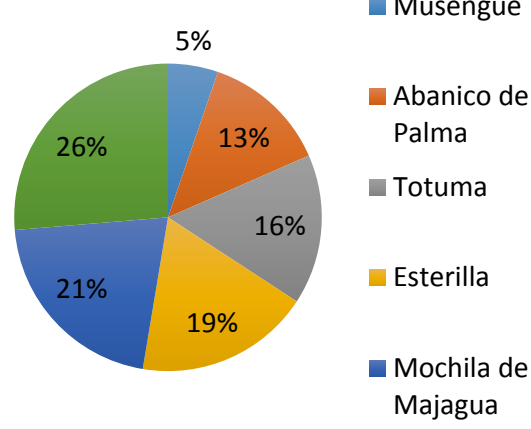

Figura 1. Conocimiento sobre artesanías.

Se indaga sobre por las diferentes artesanías, se ubicó que $26 \%$ la escoba de paja como la más conocida, seguido con $21 \%$ la mochila de majagua mientras el musengue con $5 \%$ es el menos conocidoentre los estudiantes.

\section{¿CON QUÉ TE SACUDES LOS MOSQUITOS?}

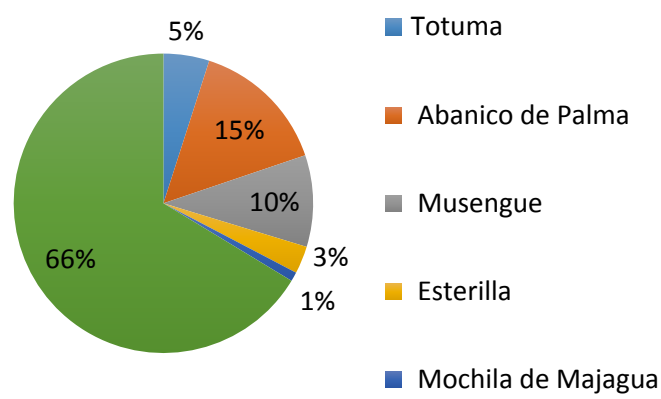

Figura 2. Funcionalidad de artesanías en palma.

En función de la información obtenida con respecto a si conocían cual era la artesanía que espantaba o mataba a los mosquitos se puede notar que la mayoría de los estudiantes desconocen para que sirvan algunas artesanías propias del pueblo, utilizando herramientas tecnológicas para dicha actividad.

\section{¿CUÁNDO BEBES CHICHA \\ DE MAÍZ EN QUE \\ RECIPIENTE LO HACEN?}

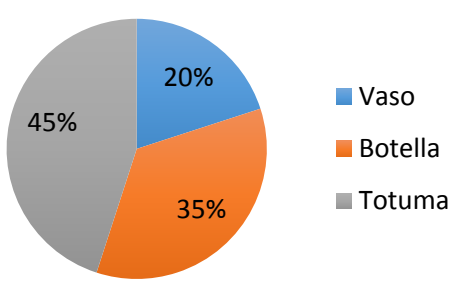

Figura 3. Funcionalidad de artesanías en totumo.

Con respecto al recipiente donde se hace una de las bebidas más comunes en el pueblo, el 55\% de los estudiantes no saben cuál es, aunque en sus casas tengan esta artesanía representativa de la caribe colombiano.

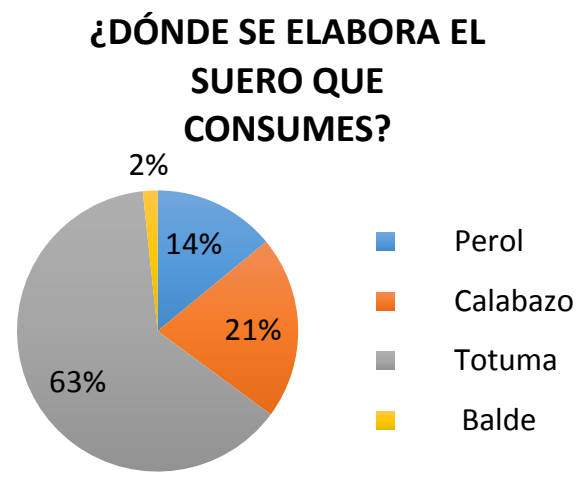

Figura 4. Artesanías en totumo.

Según el grafico anterior los estudiantes conocen el recipiente donde se realiza el suero teniendo un $63 \%$, manteniendo la tradición y costumbre, aunque hay un $37 \%$ que desconoce las utilidades de esta artesanía. 


\section{¿CON QUE MATERIAL SE ELABORA EL MUSENGUE?}

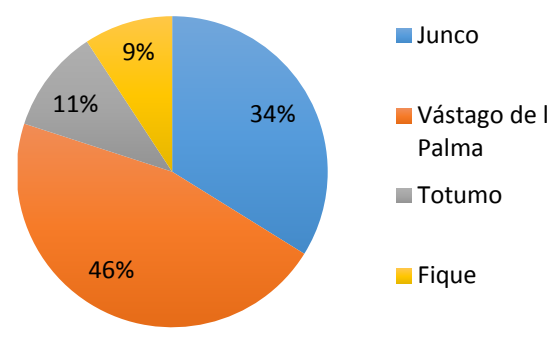

Figura 5. Artesanías en vástago de la palma.

De acuerdo con la información obtenida, el mayor porcentaje es el vástago de la palma con $46 \%$ seguido del junco con $34 \%$, teniendo esta información podemos observar que son menos de la mitad de los estudiantes que saben cómo se elabora una de las artesanías más reconocida como parte de nuestra cultura.

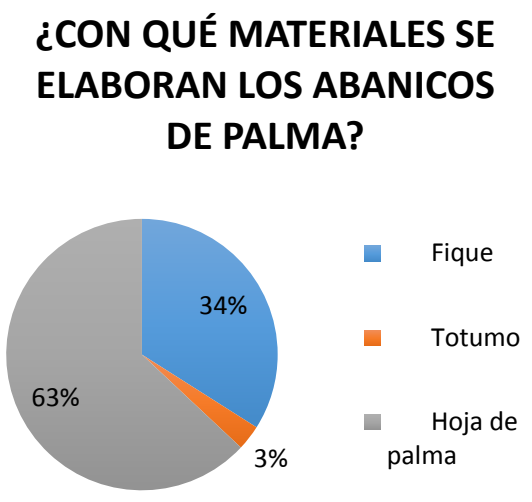

Figura 6. Artesanias en hoja de palma.

La información del gráfico podemos notar que la mayoría de fue a la hoja de palma con un $63 \%$ como segundo está el fique con $34 \%$ y tercero el totumo con un $3 \%$, los estudiantes aun no identifican las plantas con los que se hacen las artesanías propias de su pueblo.

\section{¿CON QUÉ MATERIAL SE ELABORA LA ESCOBA DE \\ PAJA?}

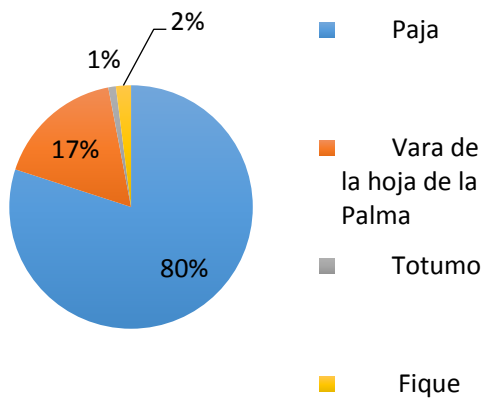

Figura 7. Materiales para elaborar escobas.

Se puede observar que la escoba una de las artesanías más conocidas y usada por el pueblo por lo cual los estudiantes reconocieron el material con el cual se realiza.

\section{¿UTILIZAS EN TU CASA ESTE TIPO DE ARTESANÍAS, CUÁLES?}

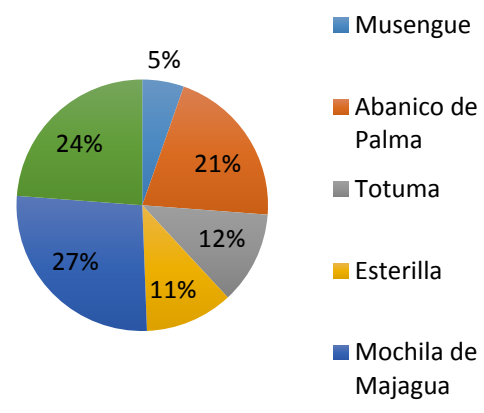

Figura 8. Indagación sobre las artesanías.

De acuerdo con lo planteado en el grafico se puede observar que la artesanía que más tienen en casa en la mochila de majagua con un $27 \%$, seguido de la escoba de paja con un $24 \%$, luego el abanico de palma con el $21 \%$, continua el totumo con el $12 \%$ después la esterilla con un $11 \%$ y finalizando con el musengue en $5 \%$. 


\section{¿SABES QUE PERSONAS \\ ELABORAN ESTAS \\ ARTESANÍAS EN NUESTRA COMUNIDAD?}

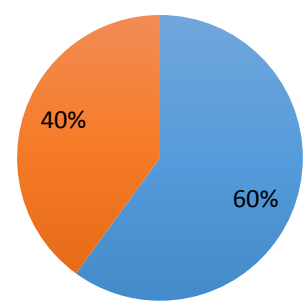

Sí

No

Figura 9. Cantidad de personas que se dedican a fabricar artesanías.

En función de la información obtenida sobre si conocen las personas que trabajan con estas artesanías la mayoría de ellos pueden identificarlas, pero aún hay ciertos estudiantes que no reconocen a estas personas.

\section{¿SABES ELABORAR ALGUNA DE ESTAS ARTESANÍAS?}

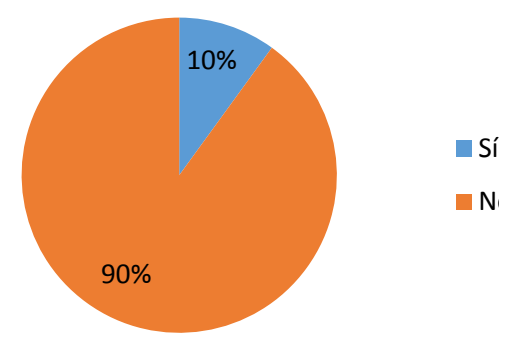

Figura 10. Conocimiento sobre elaboración de artesanías.

De acuerdo con el grafico, aunque las personas puedan reconocer las personas que realizan estas actividades no saben cómo se realizan cada una de ellas.

Nivel de conocimiento sobre conocen la esterilla los estudiantes saben que es y cómo utilizarla dada su importancia se indaga.

\section{¿CONOCES LA ESTERILLA?}

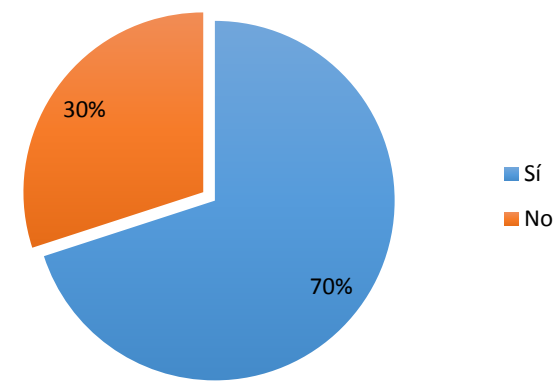

Figura 11. Conocimiento sobre la estrellita.

\section{Discusión}

Dando respuesta al objetivo de investigación, se concluye que las artesanías son una alta fuente de ingresos de las familias de los estudiantes y se logra fortalecer a nivel cultural las tradiciones de la población.

La inclusión de las TIC y EP en el cuidado y preservación de las artesanías, aporta a la economía regional y a las inclusión de las nuevas generaciones en el aprendizaje de técnicas innovadoras en términos artesanales. Se busca también el reconocimiento de la comunidad y el valor del trabajo artesanal.

\section{Referencias}

Grasso, L. (2006). Encuestas: Elementos para su diseño y análisis. Córdoba: Encuentro.

López, M. (Julio 21, 2011). Elaboración de escobas artesanales con varita de palma de vino. [Blog]. Recuperado de http://martolopez.blogspot.com.co/2011/07/elaboracion-de-escobas-artesanales-con.html

Medina, D. (2012). Propuesta para el rescate de la identidad de las técnicas ancestrales de la elaboración de las artesanías de la comunidad Ilincho. [Tesis grado]. Universidad Nacional de Loja. Loja.

Tamayoy, M. (1997). El proceso de la investigación científica. México, D.F.: Limusa.

UNESCO. (2003). Salvaguardar el patrimonio vivo de las comunidades. Recuperado de http://www.unesco.org 